\title{
The asymptotic expansion of the swallowtail integral in the highly oscillatory region
}

\author{
Chelo Ferreira ${ }^{1}$, José L. López ${ }^{2}$ and Ester Pérez Sinusía ${ }^{1}$ \\ ${ }^{1}$ Dpto. de Matemática Aplicada, IUMA, Universidad de Zaragoza \\ e-mail: cferrei@unizar.es, ester.perez@unizar.es \\ ${ }^{2}$ Dpto. de Ingeniería Matemática e Informática, Universidad Pública de Navarra and INAMAT \\ e-mail: jl.lopez@unavarra.es
}

\begin{abstract}
We consider the swallowtail integral $\Psi(x, y, z):=\int_{-\infty}^{\infty} \mathrm{e}^{\mathrm{i}\left(t^{5}+x t^{3}+y t^{2}+z t\right)} \mathrm{d} t$ for large values of $|x|$ and bounded values of $|y|$ and $|z|$. The integrand of the swallowtail integral oscillates wildly in this region and the asymptotic analysis is subtle. The standard saddle point method is complicated and then we use the simplified saddle point method introduced in [López et al., 2009]. The analysis is more straightforward with this method and it is possible to derive complete asymptotic expansions of $\Psi(x, y, z)$ for large $|x|$ and fixed $y$ and $z$. The asymptotic analysis requires the study of three different regions for $\arg x$ separated by three Stokes lines. The expansion is given in terms of inverse powers of $x^{\frac{1}{3}}$ and $x^{\frac{1}{2}}$ and the coefficients are elementary functions of $y$ and $z$. The accuracy and the asymptotic character of the approximations is illustrated with some numerical experiments.
\end{abstract}

2010 AMS Mathematics Subject Classification: 33E20; 41A60.

Keywords \& Phrases: Swallowtail integral. Asymptotic expansions. Modified saddle point method.

\section{Introduction}

The mathematical models of many short wavelength phenomena, specially wave propagation and optical diffraction, contain, as a basic ingredient, oscillatory integrals with several nearly coincident stationary phase or saddle points. The uniform approximation of those integrals 
can be expressed in terms of certain canonical integrals and their derivatives [2], [16]. The importance of these canonical diffraction integrals is stressed in [14] by means of the following sentence: The role played by these canonical diffraction integrals in the analysis of caustic wave fields is analogous to that played by complex exponentials in plane wave theory.

Apart from their mathematical importance in the uniform asymptotic approximation of oscillatory integrals [12, the canonical diffraction integrals have physical applications in the description of surface gravity waves [11], [17], bifurcation sets, optics, quantum mechanics, chemical physics [4] and acoustics (see [1, Sec. 36.14] and references there in). To our knowledge, the first application of this family of integrals traces back to the description of the disturbances on a water surface produced, for example, by a traveling ship. These disturbances form a familiar pattern of bow and stern waves which was first explained mathematically by Lord Kelvin [10] using these integrals.

In [1, Chap. 36] we can find a large amount of information about these integrals. First of all, they are classified according to the number of free independent parameters that describe the type of singularities arising in catastrophe theory, that also corresponds to the number of saddle points of the integral. The simplest integral with only one free parameter, that corresponds to the fold catastrophe, involves two coalescing stationary points: the well-known integral representation of the Airy function [13]. The second one, the Pearcey integral, depending on two free parameters, corresponds to the cusp catastrophe and involves three coalescing stationary points. The third one, depending on three free parameters corresponds to the swallowtail catastrophe and involves four coalescing stationary points. The canonical form of the oscillatory integral describing the swallowtail diffraction catastrophe is given by the swallowtail catastrophe integral [1, eq. 36.2.4]:

$$
\Psi(x, y, z):=\int_{-\infty}^{\infty} \mathrm{e}^{\mathrm{i}\left(t^{5}+x t^{3}+y t^{2}+z t\right)} \mathrm{d} t
$$

This integral exists only for $0<\arg y<\pi$ and real $x$; or for real $x, y$ and $z$.

Apart from the classification of this family of integrals, in [1, Chap. 36] we can find many properties such as symmetries, illustrative pictures, bifurcation sets, scaling relations, zeros, convergent series expansions, differential equations and leading-order asymptotic approximations among others. For example, the swallowtail integral (1) is a solution of the differential equation [1, eq. 36.10.5],

$$
\frac{\partial^{4} \Psi(x, y, z)}{\partial z^{4}}-\frac{3 x}{5} \frac{\partial^{2} \Psi(x, y, z)}{\partial z^{2}}-\mathrm{i} \frac{2 y}{5} \frac{\partial \Psi(x, y, z)}{\partial z}+\frac{z}{5} \Psi(x, y, z)=0 .
$$

On the other hand, we could not find complete asymptotic expansions of (1) in the literature.

The three first canonical integrals: Airy function, Pearcy integral and swallowtail integral are the most important ones in applications. The first one is well-known and has been deeply investigated in the literature. The second one has been considered in recent works [7, 8] and other more classical works [5, 14, 15]. In this paper we focus our attention on the third one. A numerical method for the evaluation of this integral may be found in [3]. In [1, eq. 36.8.1] 
we can find the convergent expansion:

$$
\Psi(x, y, z)=\frac{2}{5} \sum_{n=0}^{\infty} \mathrm{i}^{n} \cos \left(\frac{\pi(4 n-1)}{10}\right) \Gamma\left(\frac{n+1}{5}\right) a_{n}(x, y, z),
$$

where $a_{0}(x, y, z)=1$ and, for $n=0,1,2, \ldots$,

$$
a_{n+1}(x, y, z)=\frac{\mathrm{i}}{n+1} \sum_{p=0}^{\min (n, 2)}(p+1) \hat{x}_{p+1} a_{n-p}(x, y, z),
$$

with $\hat{x}_{1}=z, \hat{x}_{2}=y, \hat{x}_{3}=x$. The convergence speed of this expansion is rather slow for moderate or large values of the variables. In [1, eq. 36.11.2] we can find the leading order approximation of $\Psi(x, y, z)$ in terms of elementary functions, but it is valid only when the stationary points of the phase function are real and distinct. In [6] we can find an asymptotic approximation of $\Psi(x, y, z)$ in terms of Pearcey integrals, valid for large negative $x$ with $y$ real, and that remains valid when $x, y, z$ are near the cusp of the caustic. In this work we derive complete asymptotic expansions of $\Psi(x, y, z)$ that produce satisfactory approximations of $\Psi(x, y, z)$ for large $|x|$ and moderate values of $y$ and $z$, and that is valid for $x, y, z$ complex.

In the following section, we analyze the saddle point features of the swallowtail integral for large $|x|$ and fixed $y$ and $z$. In Section 3 we use a simplification of the saddle point method proposed in [9] to derive a complete asymptotic expansion of $\Psi(x, y, z)$ for large $|x|$. Section 4 contains a summary of the discussion and some numerical experiments. We use the principal argument $\arg w \in(-\pi, \pi]$ for any complex number $w$ and the notation $w^{*}$ for the complex conjugate of $w$.

\section{Preliminaries}

After splitting the integral at $t=0$ and rotating the integration interval $(-\infty, 0]$ an angle $-\frac{\pi}{10}$, and the integration interval $[0, \infty)$ an angle $\frac{\pi}{10}$, the swallowtail integral may be written in the form

$$
\Psi(x, y, z)=e^{-\mathrm{i} \frac{\pi}{10}} S\left(x \mathrm{e}^{-\mathrm{i} \frac{4 \pi}{5}}, y \mathrm{e}^{\mathrm{i} \frac{3 \pi}{10}}, z \mathrm{e}^{-\mathrm{i} \frac{3 \pi}{5}}\right)+\mathrm{e}^{\mathrm{i} \frac{\pi}{10}} S\left(x \mathrm{e}^{\mathrm{i} \frac{4 \pi}{5}}, y \mathrm{e}^{\mathrm{i} \frac{7 \pi}{10}}, z \mathrm{e}^{\mathrm{i} \frac{3 \pi}{5}}\right)
$$

with

$$
S(x, y, z):=\int_{0}^{\infty} \mathrm{e}^{-t^{5}+x t^{3}+y t^{2}+z t} \mathrm{~d} t .
$$

This last integral is absolutely convergent for all complex values of $x, y$ and $z$. Therefore, the right hand side of (4) represents the analytic continuation of the swallowtail integral $\Psi(x, y, z)$ to all complex values of $x, y$ and $z$. Then, it is more convenient to work with the representation (4)-(5) of the swallowtail integral. 


\section{The saddle point analysis of the integral $S(x, y, z)$}

\subsection{Saddle points and steepest descent paths of $S$}

Define $\theta:=\arg x$. After the change of variable $u=t \sqrt{\frac{3|x|}{5}}$ in the integral $(5)$ we find that

$$
S(x, y, z)=\sqrt{\frac{3|x|}{5}} \int_{0}^{\infty} \mathrm{e}^{\left(\frac{3|x|^{2}}{5}\right) \sqrt{\frac{3|x|}{5}} f(t)+\frac{3 y|x|}{5} t^{2}+z \sqrt{\frac{3|x|}{5}} t} \mathrm{~d} t
$$

where the phase function is $f(t):=\mathrm{e}^{\mathrm{i} \theta} t^{3}-\frac{3}{5} t^{5}$. This phase function has three saddle points: $t_{0}:=0$ and $t_{ \pm}:= \pm \mathrm{e}^{\mathrm{i} \frac{\theta}{2}}$. From the steepest descent method [18, Chap. 2], or its simplified modification [9], we know that the asymptotically relevant saddle points are those ones for which the integration path $C:=[0, \infty)$ in (6) can be deformed into a steepest descent path (or union of steepest descent paths) that contains the relevant saddle points. The point $t_{-}$ is located in the half plane $\Re(t)<0$ and has not any influence in the analysis; then we only must consider the saddle points $t_{0}$ and $t_{+}$(see Figure 1 ). Among these two points, the most relevant one is the one for which $\Re[f(t)]$ is maximal at the saddle points; and this depends on $\theta: \Re[f(0)]=0$ and $\Re\left[f\left(\mathrm{e}^{\mathrm{i} \frac{\theta}{2}}\right)\right]=\frac{2}{5} \cos \left(\frac{5 \theta}{2}\right)$. Therefore, $t_{0}$ is the relevant saddle point for $|\theta|>\frac{\pi}{5}, t_{+}$is the relevant saddle point for $|\theta|<\frac{\pi}{5}$ and both are equally relevant for $\theta=\frac{\pi}{5}$.

The steepest descent paths of $f(t)$ at the saddle points are not easy to find. On the other hand, we know from [9] that the asymptotic analysis of the integral (6) does not require the steepest descent paths of $f(t)$ at the relevant saddle points $t_{0}$ or $t_{+}$, but the steepest descent paths of the "main part" of $f(t)$ at the relevant saddle points, that may be always computed in a straightforward manner [9].

Following the notation of [9], at each saddle point $T=t_{0}$ or $T=t_{+}$, we denote by $m$ the order of the first non-vanishing derivative of $f(t)$ at the saddle point, $\phi$ is the phase of $f^{(m)}(T)$ and $f_{m}(t)$ is the Taylor polynomial of degree $m$ of $f(t)$ at the saddle point: $f_{m}(t):=f(T)+\frac{1}{m !} f^{(m)}(T)(t-T)^{m}$. The "main part" of $f(t)$ is just $f_{m}(t)$ and, at each saddle point we have that the steepest descents of $f_{m}(t)$ are the following half-straights whose end point is the saddle point $T$ [9]:

$$
\Gamma_{k}=\left\{T+r \mathrm{e}^{\mathrm{i} \theta_{k}} ; \quad \theta_{k}=\frac{(2 k+1) \pi-\phi}{m} ; r \geq 0\right\}, \quad k=0,1,2, \ldots, m-1 .
$$

Then, the first point of the asymptotic analysis of $(6)$ is the computation, at each relevant saddle point $t_{0}$ and $t_{+}$, of the steepest descent paths of $f_{m}(t)$ :

- At the saddle point $t_{0}=0$ we have that $m=3, \phi=\theta, f_{3}(t)=\mathrm{e}^{\mathrm{i} \theta} t^{3}$ and its steepest descent paths are the following three half-straights (see Figure 1):

$$
\Gamma_{k}^{0}:=\left\{r \mathrm{e}^{\mathrm{i} \theta_{k}^{0}} ; \quad \theta_{k}^{0}:=\frac{(2 k+1) \pi-\theta}{3} ; r \geq 0\right\}, \quad k=0,1,2 .
$$


- At the saddle point $t_{+}:=\mathrm{e}^{\mathrm{i} \frac{\theta}{2}}$ we have that $m=2, \phi=\pi+\frac{3 \theta}{2}, f_{2}(t)=\frac{2}{5} \mathrm{e}^{\mathrm{i} \frac{\mathrm{i} \theta}{2}}-3 \mathrm{e}^{\mathrm{i} \frac{3 \theta}{2}}(t-$ $\left.\mathrm{e}^{\mathrm{i} \frac{\theta}{2}}\right)^{2}$ and its steepest descent paths are the following two half-straights (see Figure 1 ):

$$
\Gamma_{k}^{+}:=\left\{\mathrm{e}^{\mathrm{i} \frac{\theta}{2}}+r \mathrm{e}^{\mathrm{i} \theta_{k}^{+}} ; \theta_{k}^{+}:=k \pi-\frac{3 \theta}{4} ; r \geq 0\right\}, \quad k=0,1
$$

\subsection{Deformation of the integration path}

Following [9], the second part of our analysis is to show that the path of integration $[0, \infty)$ in (6) may be deformed to a path $\Gamma$ of the form $\Gamma \equiv \bar{\Gamma} \cup \Gamma_{\epsilon}$ :

$$
S(x, y, z) \sim \sqrt{\frac{3|x|}{5}} \int_{\Gamma} \mathrm{e}^{\left(\frac{3|x|^{2}}{5}\right) \sqrt{\frac{3|x|}{5}} f(t)+\frac{3 y|x|}{5} t^{2}+z \sqrt{\frac{3|x|}{5}} t} \mathrm{~d} t .
$$

In this formula, $\bar{\Gamma}$ is one piece or the union of several pieces of the tree half-straights $\Gamma_{k}^{0}$, $k=0,1,2$, and/or of the two half-straights $\Gamma_{k}^{+}, k=0,1$, that contain(s) the most relevant saddle point $t_{0}$ or $t_{+} . \Gamma_{\epsilon}$ is an "irrelevant" path that is necessary to complete the deformation of $[0, \infty)$, but such that the integral over this path is exponentially small compared with the integral over the path $\bar{\Gamma}$. The analysis of the deformation depends on $\theta$ (see Figure 2) and all the deformations mentioned below are justified by the use of Cauchy's residue theorem:

- When $\frac{7 \pi}{10}<\theta \leq \pi$ we have that $\left|\theta_{0}^{0}\right|=\left|\frac{\pi-\theta}{3}\right|<\frac{\pi}{10}$. We can deform the path $[0, \infty)$ to the path $\Gamma_{0}^{0}$, whose end point is the relevant saddle point $t_{0}=0$. We have that $\bar{\Gamma}=\Gamma_{0}^{0}$, $\Gamma_{\epsilon}$ is empty and $\Gamma=\Gamma_{0}^{0}$ (see Figure $2(\mathrm{a})$ ).

- When $\frac{2 \pi}{5} \leq \theta<\frac{7 \pi}{10}$ we can deform the path $[0, \infty)$ to the path $\Gamma=\bar{\Gamma} \cup \Gamma_{\epsilon}$, where $\bar{\Gamma}$ is the segment $\overline{0 A}$ of $\Gamma_{0}^{0}$ plus the segment $\overline{A B}$ of $\Gamma_{0}^{+}$, where $A:=\Gamma_{0}^{0} \cup \Gamma_{0}^{+}$and $B:=\Gamma_{0}^{+} \cup[0, \infty)$. See Figure 2(b) for $\frac{2 \pi}{5}<\theta<\frac{7 \pi}{10}$ and Figure $2(\mathrm{c})$ for $\theta=\frac{2 \pi}{5}$. $\Gamma_{\epsilon}$ is the interval $[B, \infty)$.

- When $0 \leq \theta<\frac{2 \pi}{5}$ we can deform the path $[0, \infty)$ to the path $\Gamma=\bar{\Gamma} \cup \Gamma_{\epsilon}$, where $\bar{\Gamma}$ is the segment $\overline{0 A}$ of $\Gamma_{0}^{0}$ plus the segment $\overline{A B}$ of $\Gamma_{0}^{+} \cup \Gamma_{1}^{+}$(see Figure $2(\mathrm{~d})$ ). In this case $A:=\Gamma_{0}^{0} \cup \Gamma_{1}^{+}$and $\Gamma_{\epsilon}$ is the interval $[B, \infty)$.

- For negative $\theta$ the analysis is similar. For $-\pi<\theta<-\frac{7 \pi}{10}$ the analysis is identical to the case $\frac{7 \pi}{10}<\theta \leq \pi$. For $-\frac{7 \pi}{10}<\theta \leq-\frac{2 \pi}{5}$ it is identical to the case $\frac{2 \pi}{5} \leq \theta<\frac{7 \pi}{10}$ replacing $\Gamma_{0}^{0}$ by $\Gamma_{2}^{0}$. For $-\frac{2 \pi}{5}<\theta \leq 0$ it is identical to the case $0 \leq \theta<\frac{2 \pi}{5}$ replacing $\Gamma_{0}^{0}$ by $\Gamma_{2}^{0}$.

\subsection{Computation of the integrals over the steepest descent paths}

The third point of our analysis is the computation of the right hand side of $(7)$ when $\Gamma$ is one of the paths described in previous subsection. We only consider $\theta \geq 0$ as the case $\theta \leq 0$ is identical replacing $\Gamma_{0}^{0}$ by $\Gamma_{2}^{0}$ in the discussion. The following three observations are essential in the analysis: 
- We know from [9] that, except for exponentially small terms, the integral over the segment $\overline{O A}$ equals the integral over the whole steepest descent path $\Gamma_{0}^{0}$. Except for exponentially small terms, the integral over the segment $\overline{A B}$ equals the integral over the whole steepest descent path $\Gamma_{0}^{+}$when $\frac{2 \pi}{5} \leq \theta<\pi$ or over $\Gamma_{0}^{+} \cup \Gamma_{1}^{+}$when $0 \leq \theta<\frac{2 \pi}{5}$.

- Regardless of the value of $\theta$, the segment $\overline{O A}$ or the whole path $\Gamma_{0}^{0}$ is present in $\bar{\Gamma}$. Besides, when $\frac{2 \pi}{5} \leq \theta<\pi$, the path $\Gamma_{0}^{+}$is also present in $\bar{\Gamma}$ and when $0 \leq \theta<\frac{2 \pi}{5}$, then both, $\Gamma_{0}^{+}$and $\Gamma_{1}^{+}$are also present. On the other hand, as we have discussed previously, $t_{0}$ is dominant for $\theta>\frac{\pi}{5}, t_{+}$is dominant for $\theta<\pi / 5$ and both are equally relevant for $\theta=\frac{\pi}{5}$.

- For $\frac{\pi}{2} \leq \theta<\pi$ we have that $\Re[f(t)]$ is a decreasing function in $[0, \infty)$ and $\Re[f(t)] \leq$ $\Re[f(0)]=0$ for $t \in[0, \infty)$. For $0 \leq \theta<\frac{\pi}{2}$ we have that the maximum of $\Re[f(t)]$ is located at $t=\sqrt{\cos \theta}$ and is a decreasing function in $[\sqrt{\cos \theta}, \infty)$. But $B \geq C:=$ $\cos \left(\frac{\theta}{2}\right) \geq \sqrt{\cos \theta}$ and then, $\Re[f(t)]$ is a decreasing function in $\Gamma_{\epsilon}=[B, \infty)$. In any steepest descent path, $\Re[f(t)]$ decreases as $t$ runs away from the saddle point and then $\Re[f(t)] \leq \Re[f(B)]<\Re\left[f\left(\mathrm{e}^{\mathrm{i} \frac{\theta}{2}}\right)\right]$ for $t \in \Gamma_{\epsilon}=[B, \infty)$.

From the above first two observations we conclude that we may consider that $\bar{\Gamma}=\Gamma_{0}^{0}$ for $\frac{\pi}{5}<\theta \leq \pi, \bar{\Gamma}=\Gamma_{2}^{0}$ for $-\pi<\theta<-\frac{\pi}{5}, \bar{\Gamma}=\Gamma_{0}^{0} \cup \Gamma_{0}^{+} \cup \Gamma_{1}^{+}$for $0 \leq \theta \leq \frac{\pi}{5}$ and $\bar{\Gamma}=\Gamma_{2}^{0} \cup \Gamma_{0}^{+} \cup \Gamma_{1}^{+}$ for $-\frac{\pi}{5} \leq \theta \leq 0$. From the third observation we conclude that the contribution of $\Gamma_{\epsilon}$ is exponentially small compared to the contribution of $\bar{\Gamma}$ for any $\theta$. Also, the integral over $\Gamma_{2}^{0}$ is formally identical to the integral over $\Gamma_{0}^{0}$. In summary,

$$
S(x, y, z) \sim \begin{cases}S_{0}(x, y, z) & \text { if } \frac{\pi}{5}<|\theta| \leq \pi \\ S_{0}(x, y, z)+S_{1}(x, y, z) & \text { if }|\theta| \leq \frac{\pi}{5}\end{cases}
$$

where

$$
\begin{aligned}
& S_{0}(x, y, z):=\sqrt{\frac{3|x|}{5}} \int_{\Gamma_{0}^{0}} \mathrm{e}^{\left(\frac{3|x|^{2}}{5}\right) \sqrt{\frac{3|x|}{5}} f_{3}(t)} \mathrm{e}^{\left(\frac{3|x|^{2}}{5}\right) \sqrt{\frac{3|x|}{5}}\left[f(t)-f_{3}(t)\right]+\frac{3 y|x|}{5} t^{2}+z \sqrt{\frac{3|x|}{5}} t} \mathrm{~d} t, \quad \theta \geq 0 \\
& S_{0}(x, y, z):=\sqrt{\frac{3|x|}{5}} \int_{\Gamma_{2}^{0}} \mathrm{e}^{\left(\frac{3|x|^{2}}{5}\right) \sqrt{\frac{3|x|}{5}} f_{3}(t)} \mathrm{e}^{\left(\frac{3|x|^{2}}{5}\right) \sqrt{\frac{3|x|}{5}}\left[f(t)-f_{3}(t)\right]+\frac{3 y|x|}{5} t^{2}+z \sqrt{\frac{3|x|}{5}} t} \mathrm{~d} t, \quad \theta \leq 0 \\
& S_{1}(x, y, z):=\sqrt{\frac{3|x|}{5}} \int_{\Gamma_{0}^{+} \cup \Gamma_{1}^{+}} \mathrm{e}^{\left(\frac{3 \mid x x^{2}}{5}\right) \sqrt{\frac{3|x|}{5}} f_{2}(t)} \mathrm{e}^{\left(\frac{3|x|^{2}}{5}\right) \sqrt{\frac{3|x|}{5}}\left[f(t)-f_{2}(t)\right]+\frac{3 y|x|}{5} t^{2}+z \sqrt{\frac{3|x|}{5}} t} \mathrm{~d} t
\end{aligned}
$$

and $f_{2}(t)$ and $f_{3}(t)$ have been defined in Section 3.1 .

As it will be clear in the next section, in the open sector $|\theta|<\frac{\pi}{5}, S_{1}(x, y, z)$ dominates $S_{0}(x, y, z)$, whereas on the lines $\arg (x)= \pm \frac{\pi}{5}$ either, $S_{1}(x, y, z)$ or $S_{0}(x, y, z)$ may be dominant 
depending on the arguments of $y$ and $z$. Therefore, instead of (8), we may write the more natural formula

$$
S(x, y, z) \sim \begin{cases}S_{0}(x, y, z) & \text { if } \frac{\pi}{5}<|\theta| \leq \pi \\ S_{0}(x, y, z)+S_{1}(x, y, z) & \text { if }|\theta|=\frac{\pi}{5} \\ S_{1}(x, y, z) & \text { if }|\theta|<\frac{\pi}{5}\end{cases}
$$

Figure 3(a) shows the two different asymptotic expansions of $S(x, y, z)$ in the two sectors of the complex $x$ plane, according to (12).

\subsection{The approximation of $S_{0}$ and $S_{1}$}

The fourth point of the analysis is the approximation of the integrals $S_{0}(x, y, z)$ and $S_{1}(x, y, z)$. For positive $\theta$, we perform the change of variable $t \rightarrow \sqrt{\frac{5}{3}} \mathrm{e}^{\mathrm{i} \frac{\pi-\theta}{3}}|x|^{-\frac{5}{6}} t$ in $(9)$. For negative $\theta$, we perform the change of variable $t \rightarrow \sqrt{\frac{5}{3}} \mathrm{e}^{-\mathrm{i} \frac{\pi+\theta}{3}}|x|^{-\frac{5}{6}} t$ in 10$)$. In any case we find

$$
S_{0}(x, y, z)=\frac{1}{(-x)^{\frac{1}{3}}} \int_{0}^{\infty} \mathrm{e}^{-t^{3}} g(x, y, z, t) \mathrm{d} t
$$

with

$$
g(x, y, z, t):=\exp \left(-\frac{t^{5}}{(-x)^{\frac{5}{3}}}+\frac{y t^{2}}{(-x)^{\frac{2}{3}}}+\frac{z t}{(-x)^{\frac{1}{3}}}\right) .
$$

From here, the computation of the asymptotic expansion of this integral follows as in Watson's Lemma [9]. Replacing $g(x, y, z, t)$ by its Taylor expansion at $t=0$, and interchanging sum and integral we find

$$
S_{0}(x, y, z) \sim \frac{1}{3} \sum_{n=0}^{\infty} \Gamma\left(\frac{n+1}{3}\right) \frac{B_{n}(y, z)}{(-x)^{\frac{n+1}{3}}} \quad \text { as } \quad|x| \rightarrow \infty,
$$

with

$$
B_{n}(y, z):=\sum_{5 k+2 j+l=n} \frac{(-1)^{k} y^{j} z^{l}}{k ! j ! l !},
$$

where the sum runs for all $k, j, l \in \mathbb{N}$ such that $5 k+2 j+l=n$. The first few coefficients $B_{n}$ are

$$
B_{0}(y, z)=1, \quad B_{1}(y, z)=z, \quad B_{2}(y, z)=y+\frac{z^{2}}{2} .
$$

The coefficients $B_{n}$ are independent of $x$. Then we have that every term in the expansion of $S(x, y, z)$ in $(15)$ is of the order $\mathcal{O}\left(x^{-\frac{n+1}{3}}\right)$ as $|x| \rightarrow \infty$ for bounded $y$ and $z$.

After the change of variables $t \rightarrow \mathrm{e}^{\mathrm{i} \frac{\theta}{2}}+\mathrm{e}^{-\mathrm{i} \frac{3 \theta}{4}}\left(\frac{5}{3}\right)^{\frac{3}{4}} \frac{t}{|x|^{\frac{5}{4}} \sqrt{3}}$ in the integral (11) we find

$$
S_{1}(x, y, z)=\frac{1}{\sqrt{5}}\left(\frac{5}{3 x}\right)^{3 / 4} \mathrm{e}^{\frac{2 x}{5}\left(\frac{3 x}{5}\right)^{3 / 2}+\frac{3 x y}{5}+z \sqrt{\frac{3 x}{5}}} \int_{-\infty}^{\infty} \mathrm{e}^{-t^{2}} g(x, y, z, t) \mathrm{d} t
$$


with

$$
g(x, y, z, t):=\exp \left\{\left(\frac{5^{\frac{1}{4}} z}{3^{\frac{3}{4}} x^{\frac{3}{4}}}+\frac{2 y}{15^{\frac{1}{4}} x^{\frac{1}{4}}}\right) t+\frac{\sqrt{5} y t^{2}}{3^{\frac{3}{2}} x^{\frac{3}{2}}}-\frac{5^{\frac{7}{4}} t^{3}}{9 \cdot 3^{\frac{1}{4}} x^{\frac{5}{4}}}-\frac{5^{\frac{3}{2}} t^{4}}{3^{\frac{5}{2}} x^{\frac{5}{2}}}-\frac{5^{\frac{5}{4}} t^{5}}{27 \cdot 3^{\frac{3}{4}} x^{\frac{15}{4}}}\right\} .
$$

Replacing the Taylor expansion of $g(x, y, z, t)$ at $t=0$, and interchanging sum and integral in the above expression, we find that, as $|x| \rightarrow \infty$,

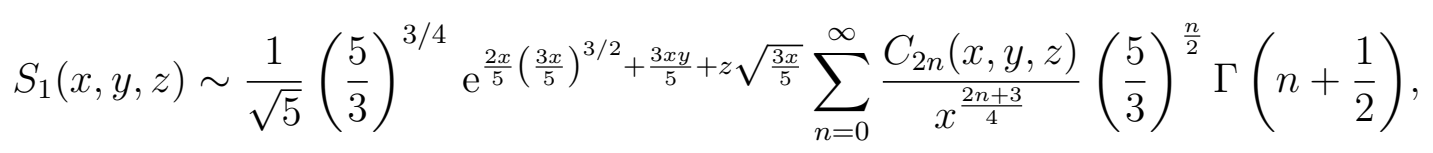

with

$$
C_{n}(x, y, z):=\sum_{5 k+4 j+3 l+2 m+s=n} \frac{(-1)^{k+j+l} 5^{\frac{j+2 l}{2}}}{7^{k} 3^{\frac{k+3 j+3 l+2 m}{2}} k ! j ! l ! m ! s !} \frac{y^{m}}{x^{\frac{5 k+3 j+l+2 m}{2}}}\left(\frac{z}{\sqrt{3 x}}+\frac{2 y}{\sqrt{5}}\right)^{s},
$$

where the sum runs for all $k, j, l, m, s \in \mathbb{N}$ such that $5 k+4 j+3 l+2 m+s=n$. The first few relevant coefficients $C_{n}$ are

$$
\begin{aligned}
C_{0}(x, y, z)= & 1, \quad C_{2}(x, y, z)=\frac{2 y^{2}}{5}+\frac{2 y z}{\sqrt{15 x}}+\frac{2 y+z^{2}}{6 x} \\
C_{4}(x, y, z)= & \frac{2 y^{4}}{75}+\frac{2 \sqrt{15} y\left(2 y^{2} z-25\right)}{225 \sqrt{x}}+\frac{6 y^{3}-25 z+3 y^{2} z^{2}}{45 x} \\
& +\frac{\sqrt{15}\left(6 y^{2} z+y z^{3}-15\right)}{135 x \sqrt{x}}+\frac{12 y^{2}+12 y z^{2}+z^{4}}{216 x^{2}} .
\end{aligned}
$$

The coefficients $C_{n}$ are bounded for $|x|$ bounded from below and fixed $y$ and $z$. Then we have that, apart from an exponential factor, every term in the expansion of $S(x, y, z)$ in (20) is of the order $\mathcal{O}\left(x^{-\frac{2 n+3}{4}}\right)$ as $|x| \rightarrow \infty$ for bounded $y$ and $z$.

\section{Summary of the discussion}

From (12), (15) and (20) we see that, when $|x| \rightarrow \infty, S(x, y, z) \sim S_{0}(x, y, z)$ is of the order $\mathcal{O}\left(x^{-\frac{1}{3}}\right)$ if $|\arg x|>\frac{\pi}{5}$ and $S(x, y, z) \sim S_{1}(x, y, z)$ is of the order $\mathcal{O}\left(x^{-\frac{3}{4}} \mathrm{e}^{\frac{2 x}{5}\left(\frac{3 x}{5}\right)^{\frac{3}{2}}}\right)$ if $|\arg x|<$ $\frac{\pi}{5}$. Then, $S(x, y, z)$ has an exponential behavior in the red region of Figure 3(a) and a power behavior in the blue and purple regions. The Stokes lines of $S(x, y, z)$ are $\arg x= \pm \frac{\pi}{5}$. Over these lines, $S(x, y, z) \sim S_{0}(x, y, z)+S_{1}(x, y, z)=\mathcal{O}\left(x^{-\frac{1}{3}}\right)+\mathcal{O}\left(x^{-\frac{3}{4}} \mathrm{e}^{\frac{2 x}{5}\left(\frac{3 x}{5}\right)^{3 / 2}+\frac{3 x y}{5}+z \sqrt{\frac{3 x}{5}}}\right)$ and then, the precise order of $S(x, y, z)$ over its Stokes lines depends on the arguments of $y$ and $z$.

To determine the asymptotic behavior of the swallowtail integral we must use (4). The function $S(x, y, z)$ is evaluated at $x \mathrm{e}^{-\mathrm{i} \frac{4 \pi}{5}}$ in the first term and at $x \mathrm{e}^{\mathrm{i} \frac{4 \pi}{5}}$ in the second one. Therefore, the Stokes lines of the swallowtail integral $\operatorname{are} \arg (x)= \pm \frac{3 \pi}{5}$ and $\arg (x)=\pi$, and 
the complex plane is divided into the three regions depicted in Figure 3(b) according to the asymptotic behavior of $\Psi(x, y, z)$ :

$$
\Psi(x, y, z) \sim \begin{cases}S_{1}^{+} & \text {for }-\pi<\arg x<-\frac{3 \pi}{5}, \\ S_{1}^{+}+S_{0}^{-}+S_{0}^{+} & \text {for } \arg x=-\frac{3 \pi}{5}, \\ S_{0}^{-}+S_{0}^{+} & \text {for }-\frac{3 \pi}{5}<\arg x<\frac{3 \pi}{5}, \\ S_{1}^{-}+S_{0}^{-}+S_{0}^{+} & \text {for } \arg x=\frac{3 \pi}{5}, \\ S_{1}^{-} & \text {for } \frac{3 \pi}{5}<\arg x<\pi, \\ S_{1}^{-}+S_{1}^{+}+S_{0}^{-}+S_{0}^{+} & \text {for } \arg x=\pi,\end{cases}
$$

where, in order to simplify the formula, we have used the notation

$$
\begin{aligned}
& S_{0}^{-}:=\mathrm{e}^{-\mathrm{i} \frac{\pi}{10}} S_{0}\left(x \mathrm{e}^{-\mathrm{i} \frac{4 \pi}{5}}, y \mathrm{e}^{\mathrm{i} \frac{3 \pi}{10}}, z \mathrm{e}^{-\mathrm{i} \frac{3 \pi}{5}}\right), \quad S_{0}^{+}:=\mathrm{e}^{\mathrm{i} \frac{\pi}{10}} S_{0}\left(x \mathrm{e}^{\mathrm{i} \frac{4 \pi}{5}}, y \mathrm{e}^{\mathrm{i} \frac{7 \pi}{10}}, z \mathrm{e}^{\mathrm{i} \frac{3 \pi}{5}}\right), \\
& S_{1}^{-}:=\mathrm{e}^{-\mathrm{i} \frac{\pi}{10}} S_{1}\left(x \mathrm{e}^{-\mathrm{i} \frac{4 \pi}{5}}, y \mathrm{e}^{\mathrm{i} \frac{3 \pi}{10}}, z \mathrm{e}^{-\mathrm{i} \frac{3 \pi}{5}}\right), \quad S_{1}^{+}:=\mathrm{e}^{\mathrm{i} \frac{\pi}{10}} S_{1}\left(x \mathrm{e}^{\mathrm{i} \frac{4 \pi}{5}}, y \mathrm{e}^{\mathrm{i} \frac{7 \pi}{10}}, z \mathrm{e}^{\mathrm{i} \frac{3 \pi}{5}}\right) .
\end{aligned}
$$

The function $S_{0}(x, y, z)$ has the asymptotic expansion given in (15) and $S_{1}(x, y, z)$ has the asymptotic expansion given in 20 . At the Stokes lines of $\Psi(x, y, z), \arg x= \pm \frac{3 \pi}{5}$ and $\arg x=\pi$, the arguments of $y$ and $z$ determine which of the terms $S_{1}^{ \pm}$or $S_{0}^{ \pm}$is dominant.

Consider $x<0$ and real $y, z$, where the approximations [1, eq. 36.11.2] and [6] are valid. From the sixth line of $(23),(24)$, and the first order approximation given by the first term of the right hand side of (15) and (20), it is straightforward to check that the first order approximations given in [1, eq. 36.11.2] and [6] are reproduced.

\section{$5 \quad$ Numerical experiments}

In Table 1 we can find some numerical experiments that show the accuracy of the approximations summarized above. We have used formula (4), with a numerical evaluation of (5), to compute the "exact" value of $\Psi(x, y, z)$. We evaluate (5) with the NIntegrate command of Wolfram Mathematica 10.4. The approximations (15)-(16) and (20)-(21) used in (23)-(24) have been computed by using this software. The relative errors referred in Table 1 and Figures 4 and 5 are defined as

$$
\left|\frac{\Psi(x, y, z)-\Psi_{n}(x, y, z)}{\Psi(x, y, z)}\right|, \quad n=1,2,3, \ldots
$$

where $\Psi_{n}(x, y, z)$ is the right hand side of $(23)$, with $S_{0}^{ \pm}$and $S_{1}^{ \pm}$replaced by the first $n$ terms of the right hand side of (15) and (20) respectively (and taking into account (24)).

\section{Acknowledgments}

This research was supported by the Ministerio de Economía y Competitividad (MTM201452859) and the Universidad Pública de Navarra. Professor Adri Olde Daalhuis is acknowledged by his helpful comments and suggestions. The anonymous referee is acknowledged 
by his/her comprehensive revision of the manuscript and his/her numerous improving suggestions.

\section{References}

[1] M. V. Berry and C. J. Howls, Integrals with coalescing saddles, in: NIST Handbook of Mathematical Functions, Cambridge University Press, Cambridge, 2010, pp. 775-793 (Chapter 36).

[2] J. N. L. Connor and P. R. Kurtis, A method for the numerical evaluation of the oscillatory integrals associated with the cuspoid catastrophes: application to Pearcey's integral and its derivatives, J. Phys. A, 15 n. 4 (1982), 1179-1190.

[3] J. N. L. Connor, P. R. Curtis, and D. Farrelly, A differential equation method for the numerical evaluation of the Airy, Pearcey and swallowtail canonical integrals and their derivatives, Molecular Physics, $48 \mathrm{n}$. 6 (1983) 1305-1330.

[4] J. N. L. Connor, P. R. Curtis, and R. A. W. Young, Uniform asymptotics of oscillating integrals: applications in chemical physics. In P. A. Martin 6 and G. R. Wickman, editors, Wave Asymptotics, chapter 2, pages 24-42. Cambridge University Press, Cambridge, 1992.

[5] D. Kaminski, Asymptotic expansion of the Pearcey integral near the caustic, J. SIAM J. Math. Anal., 20 n. 4 (1989), 987-1005.

[6] D. Kaminski, Asymptotics of the swallowtail integral near the cusp of the caustic, J. SIAM J. Math. Anal., 23 n. 1 (1992), 262-285.

[7] J. L. López and P. Pagola, Convergent and asymptotic expansions of the Pearcey integral, J. Math. Anal. Appl., 430 n. 1 (2015) 181-192.

[8] J. L. López and P. Pagola, The Pearcy integral in the highly oscillatory region, Appl. Math. Comput., 275 (2016) 404-410.

[9] J. L. López, E. Pérez Sinusía and P. Pagola, A systematization of the saddle point method. Application to the Airy and Hankel functions, J. Math. Anal. Appl., 354 (2009), 347-359.

[10] Lord Kelvin, Popular Lectures and Addresses, Vol. 3, London: Macmillan, (1891), 481-488.

[11] Lord Kelvin, Deep water ship-waves, Phil. Mag., 9 (1905), 733-757.

[12] A. B. Olde Daalhuis, On the asymptotics for late coefficients in uniform asymptotic expansions of integrals with coalescing saddles Meth. Appl. Anal., 7 no. 4 (2000), 727-745.

[13] F. W. J. Olver, Airy and related functions, in: NIST Handbook of Mathematical Functions, Cambridge University Press, Cambridge, 2010, pp. 193-214 (Chapter 9).

[14] R. B. Paris, The asymptotic behaviour of Pearcey's integral for complex variables, Proc. Roy. Soc. London Ser. A., 432 no. 1886 (1991), 391-426.

[15] R. B. Paris and D. Kaminski, Hyperasymptotic evaluation of the Pearcey integral via Hadamard expansions, J. Comput. Appl. Math., 190 (2006), n. 1-2, 437-452.

[16] F. Ursell, Integrals with a large parameter: several nearly coincident saddle points, Proc. Camb. Phil. Soc., 72 (1972), 49-65.

[17] F. Ursell, Ship Hydrodynamics, Water Waves and Asymptotics, Vol. 2 of Collected works of F. Ursell, 1946-1992. Singapore: World Scientific, 1994.

[18] R. Wong, Asymptotic approximations of integrals, Academic Press, New York, 1989. 


\begin{tabular}{|c|c|c|c|c|c|c|c|}
\hline \multicolumn{4}{|c}{$y=0.5 \mathrm{e}^{\mathrm{i} \frac{\pi}{6}}, z=0.25 \mathrm{i}$} & \multicolumn{1}{c|}{$y=0.25, z=3.1$} \\
\hline$x$ & $n=1$ & $n=3$ & $n=5$ & $x$ & $n=2$ & $n=4$ & $n=6$ \\
\hline 10 & 0.0066 & 0.0035 & 0.0023 & -5 & 0.3 & 0.08 & 0.029 \\
\hline 50 & $1 . \mathrm{e}-3$ & $2.7 \mathrm{e}-4$ & $7.3 \mathrm{e}-5$ & -10 & 0.1 & 0.015 & 0.0042 \\
\hline 100 & $5 . \mathrm{e}-4$ & $9.7 \mathrm{e}-4$ & $1.7 \mathrm{e}-5$ & -40 & 0.03 & 0.001 & $3 . \mathrm{e}-4$ \\
\hline
\end{tabular}

\begin{tabular}{|c|c|c|c|c|c|c|c|}
\multicolumn{4}{c|}{$y=0.25 \mathrm{e}^{\mathrm{i} \frac{\pi}{5}}, z=0.1$} & \multicolumn{5}{c|}{$y=-0.2, z=0.3 \mathrm{i}$} \\
\hline$x$ & $n=1$ & $n=3$ & $n=5$ & $x$ & $n=2$ & $n=4$ & $n=6$ \\
\hline $5 \mathrm{e}^{\mathrm{i} \frac{\pi}{4}}$ & 0.006 & 0.007 & 0.006 & $5 \mathrm{e}^{\mathrm{i} \frac{4 \pi}{5}}$ & 0.035 & 0.006 & $2.4 \mathrm{e}-4$ \\
\hline $10 \mathrm{e}^{\mathrm{i} \frac{\pi}{4}}$ & 0.0014 & 0.00138 & $8.7 \mathrm{e}-4$ & $10 \mathrm{e}^{\mathrm{i} \frac{4 \pi}{5}}$ & 0.0014 & 0.00138 & $8.7 \mathrm{e}-4$ \\
\hline $100 \mathrm{e}^{\mathrm{i} \frac{\pi}{4}}$ & $8.2 \mathrm{e}-5$ & $2.6 \mathrm{e}-5$ & $4.3 \mathrm{e}-6$ & $50 \mathrm{e}^{\mathrm{i} \frac{4 \pi}{5}}$ & $1 . \mathrm{e}-4$ & $4.4 \mathrm{e}-7$ & $2.9 \mathrm{e}-9$ \\
\hline
\end{tabular}

\begin{tabular}{|c|c|c|c|c|c|c|c|}
\hline \multicolumn{1}{c|}{$y=0.5 \mathrm{i}, z=-0.5$} & \multicolumn{5}{c|}{$y=1.2 \mathrm{e}^{-\mathrm{i} \frac{\pi}{3}}, z=2.1 \mathrm{e}^{\mathrm{i} \frac{\pi}{4}}$} \\
\hline$x$ & $n=2$ & $n=4$ & $n=6$ & $x$ & $n=2$ & $n=4$ & $n=6$ \\
\hline $4 \mathrm{e}^{-\mathrm{i} \frac{4 \pi}{5}}$ & 0.07 & 0.05 & 0.02 & $5 \mathrm{e}^{-\mathrm{i} \frac{\pi}{6}}$ & 0.17 & 0.06 & 0.05 \\
\hline $10 \mathrm{e}^{-\mathrm{i} \frac{4 \pi}{5}}$ & 0.0056 & $7.8 \mathrm{e}-4$ & $7.5 \mathrm{e}-5$ & $50 \mathrm{e}^{-\mathrm{i} \frac{\pi}{6}}$ & 0.017 & $5 . \mathrm{e}-4$ & $1.6 \mathrm{e}-4$ \\
\hline $60 \mathrm{e}^{-\mathrm{i} \frac{4 \pi}{5}}$ & $6 . \mathrm{e}-5$ & $5.3 \mathrm{e}-7$ & $1.2 \mathrm{e}-8$ & $500 \mathrm{e}^{-\mathrm{i} \frac{\pi}{6}}$ & 0.0016 & $4.8 \mathrm{e}-6$ & $6.3 \mathrm{e}-7$ \\
\hline
\end{tabular}

\begin{tabular}{|c|c|c|c||c|c|c|c|}
\hline \multicolumn{1}{|c|}{$y=0.4 \mathrm{e}^{-\mathrm{i} \frac{\pi}{12}}, z=1.1$} & \multicolumn{5}{c|}{$y=-02 \mathrm{i}, z=0.1 \mathrm{e}^{\mathrm{i} \frac{\pi}{6}}$} \\
\hline$x$ & $n=2$ & $n=4$ & $n=6$ & $x$ & $n=2$ & $n=4$ & $n=6$ \\
\hline $30 \mathrm{e}^{-\mathrm{i} \frac{3 \pi}{5}}$ & 0.004 & $5 . \mathrm{e}-3$ & $1 . \mathrm{e}-3$ & $10 \mathrm{e}^{\mathrm{i} \frac{3 \pi}{5}}$ & $9.4 \mathrm{e}-4$ & $8.6 \mathrm{e}-4$ & $3.7 \mathrm{e}-4$ \\
\hline $70 \mathrm{e}^{-\mathrm{i} \frac{3 \pi}{5}}$ & 0.002 & $8.3 \mathrm{e}-5$ & $2.5 \mathrm{e}-5$ & $50 \mathrm{e}^{\mathrm{i} \frac{3 \pi}{5}}$ & $2.9 \mathrm{e}-5$ & $1.7 \mathrm{e}-5$ & $1.8 \mathrm{e}-6$ \\
\hline $150 \mathrm{e}^{-\mathrm{i} \frac{3 \pi}{5}}$ & $9.5 \mathrm{e} .4$ & $2 . \mathrm{e}-5$ & $3 . \mathrm{e}-6$ & $100 \mathrm{e}^{\mathrm{i} \frac{3 \pi}{5}}$ & $1 . \mathrm{e}-5$ & $4.15 \mathrm{e}-6$ & $2.5 \mathrm{e}-7$ \\
\hline
\end{tabular}

Table 1: Numerical experiments about the relative errors for several approximations of the integral $\Psi(x, y, z)$ given in (1) and different complex values of $x, y$ and $z$, in different regions of Figure 3(b), using formulas (23) and 24. 


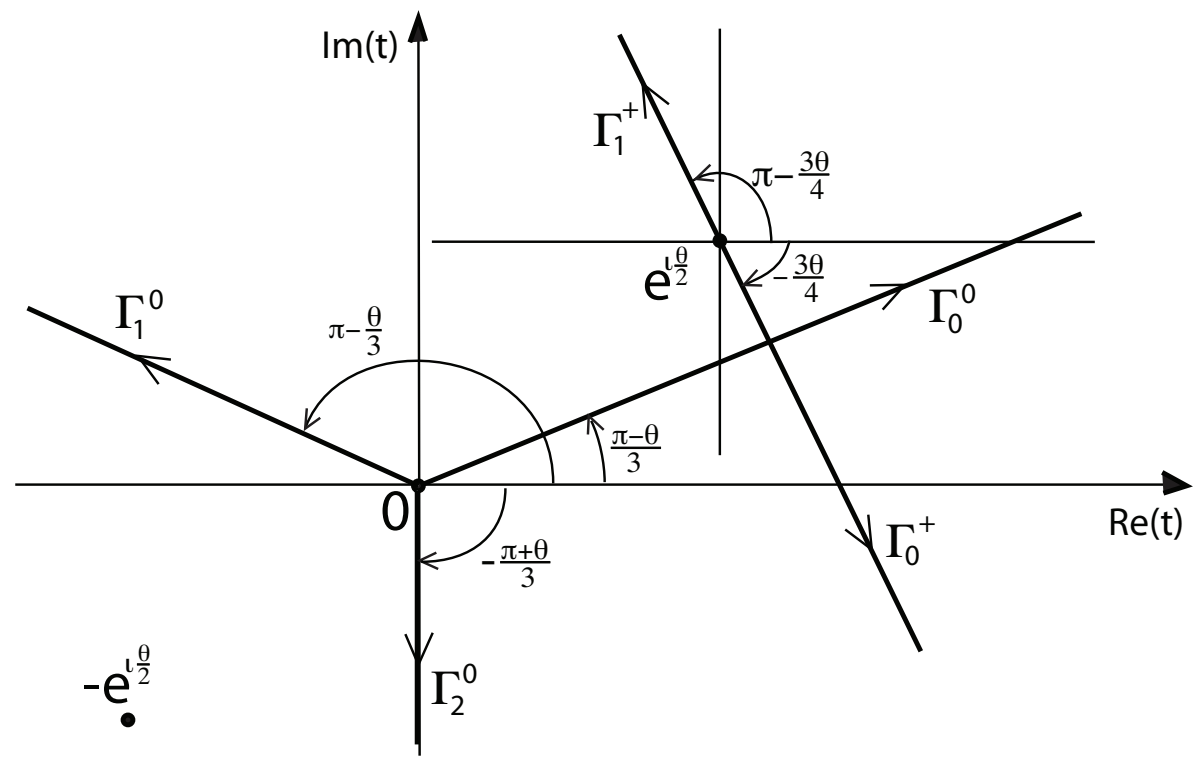

Figure 1: This picture corresponds to $\theta=\frac{\pi}{2}$. Saddle points $t_{0}:=0$ and $t_{ \pm}:= \pm \mathrm{e}^{\mathrm{i} \frac{\theta}{2}}$ of the phase function in (6) and the steepest descent paths at 0 and $\mathrm{e}^{\mathrm{i} \frac{\theta}{2}}$. The integration path $[0, \infty)$ in $(6)$ can be deformed to a steepest descent path at the point 0 or to the union of a steepest descent path at 0 and another steepest descent path at $\mathrm{e}^{\mathrm{i} \frac{\theta}{2}}$. Therefore, the point $-\mathrm{e}^{\mathrm{i} \frac{\theta}{2}}$ has not any influence in the asymptotic analysis of (6). 


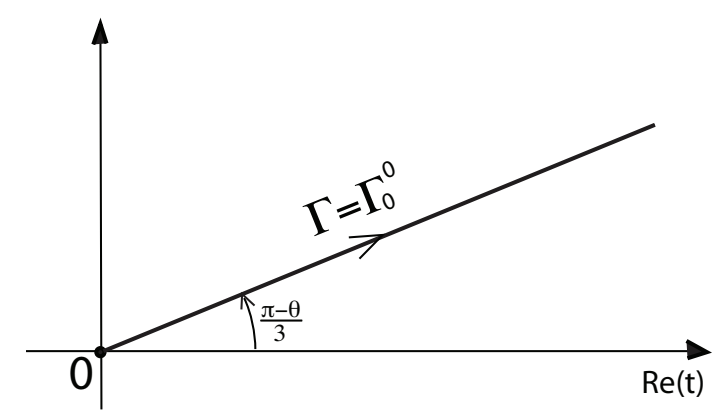

(a)

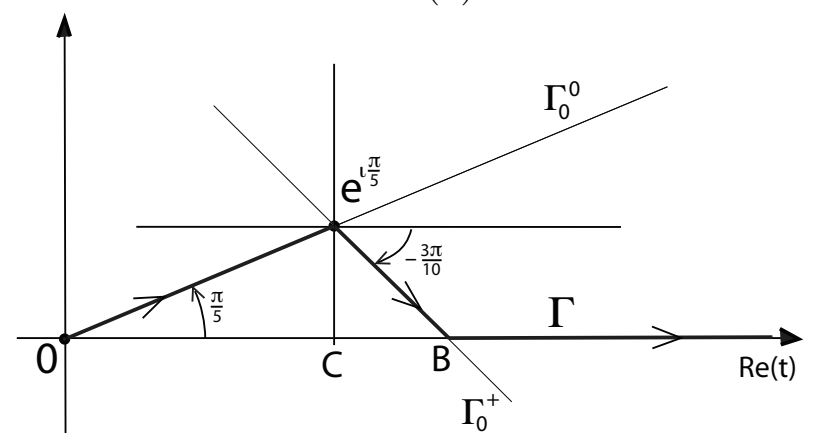

(c)

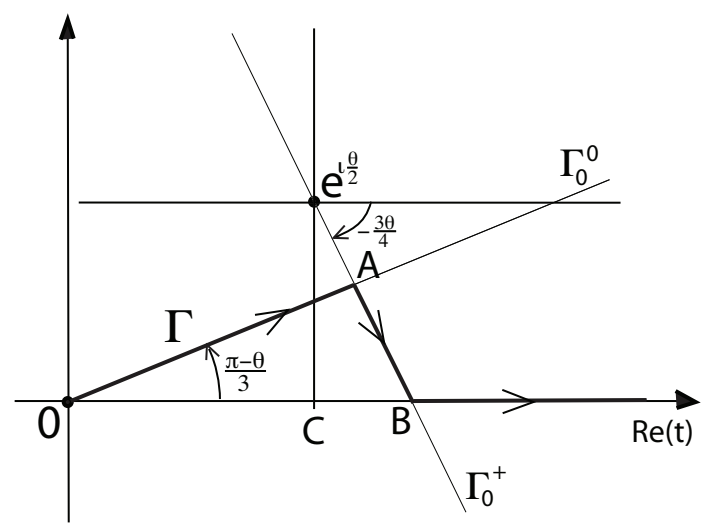

(b)

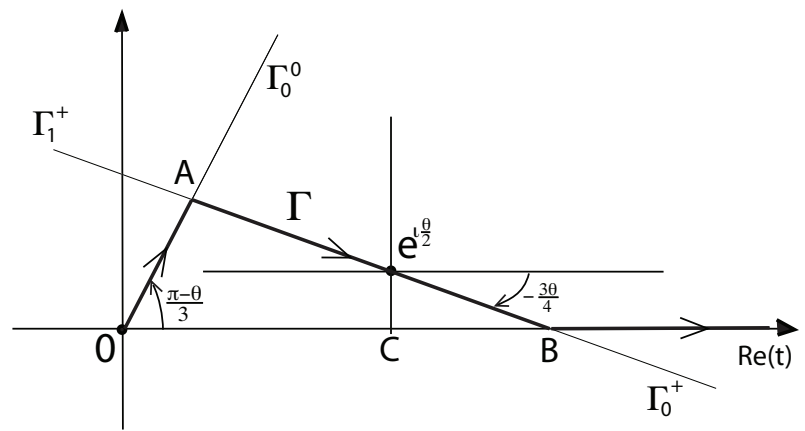

(d)

Figure 2: (a) When $\frac{7 \pi}{10}<\theta \leq \pi$ the path $[0, \infty)$ is deformed to the path $\Gamma=\Gamma_{0}^{0}$. When $\frac{2 \pi}{5} \leq \theta<\pi$ the path $[0, \infty)$ is deformed to the path $\Gamma=\overline{O A} \cup \overline{A B} \cup[B, \infty)$ represented in Figure (b) for $\frac{2 \pi}{5} \leq \theta<\pi$ or in Figure (c) for $\theta=\frac{2 \pi}{5}$ (when $\theta=\frac{2 \pi}{5}$ then $A=t_{+}=\mathrm{e}^{\mathrm{i} \frac{\theta}{2}}$ ). (d) When $0 \leq \theta<\frac{2 \pi}{5}$ the path $[0, \infty)$ is deformed to the path $\Gamma=\overline{O A} \cup \overline{A B} \cup[B, \infty)$. 


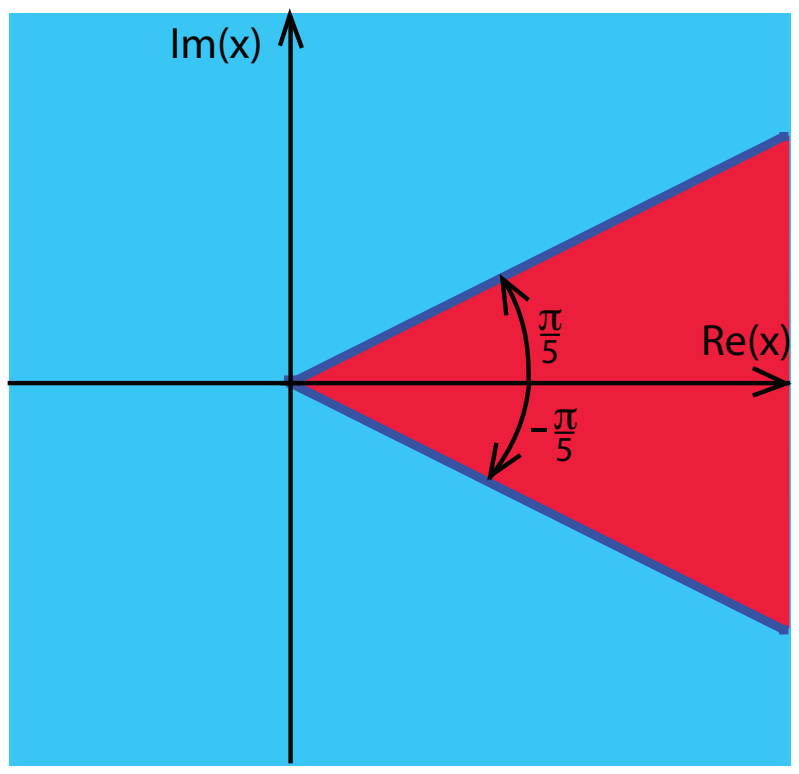

(a)

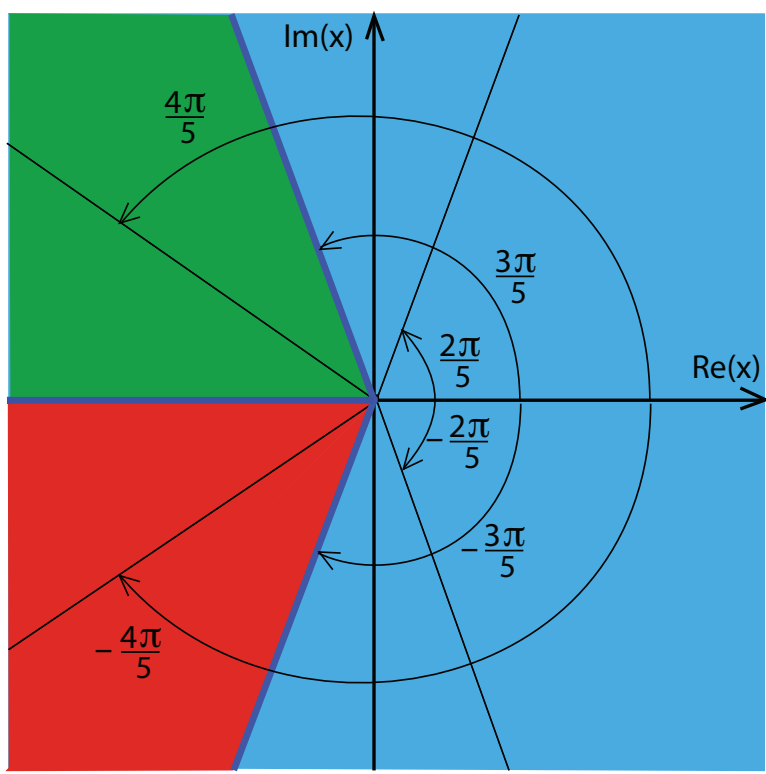

(b)

Figure 3: (a) The saddle point analysis of the integral (5) is different in the two regions of the complex $x$ plane separated by lines $\arg x= \pm \frac{\pi}{5}$. (b) The asymptotic behavior of the swallowtail integral is different in the three regions depicted in the figure; the Stokes lines $\operatorname{are} \arg (x)= \pm \frac{3 \pi}{5}$ and $\arg (x)=\pi$.
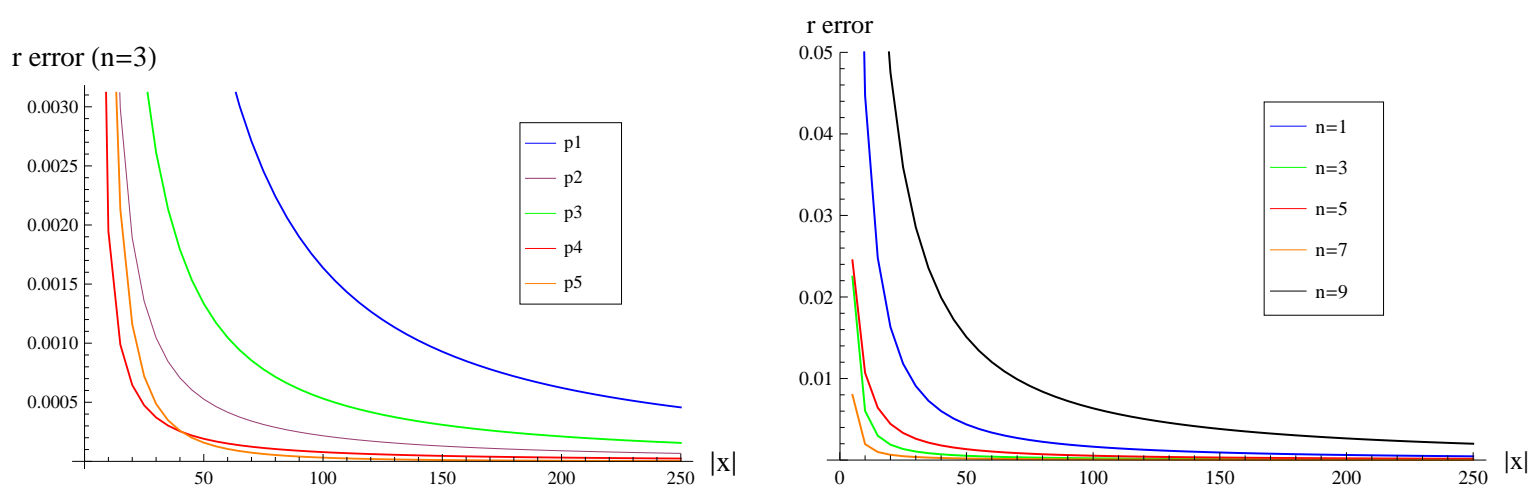

Figure 4: Left picture: relative errors for approximation order $n=3$; five different values of the pair $(y, z): p 1\left(1.2 e^{-\mathrm{i} \pi / 3}, 2.1 e^{\mathrm{i} \pi / 4}\right), p 2(0.5,-1.2), p 3(1.2,0.15), p 4\left(0.5 e^{\mathrm{i} \pi / 4},-0.2 e^{\mathrm{i} \pi / 4}\right)$, $p 5(-0.2 \mathrm{i},-1.2)$ and $0<|x|<250$, with $\arg (x)=-\pi / 6$. Right picture: relative errors for five different values of the approximation degree $n$ and $0<|x|<250$, with $\arg (x)=\pi / 6$, $y=1.2 e^{-\mathrm{i} \pi / 3}$ and $z=0.3 e^{i \pi / 4}$. 

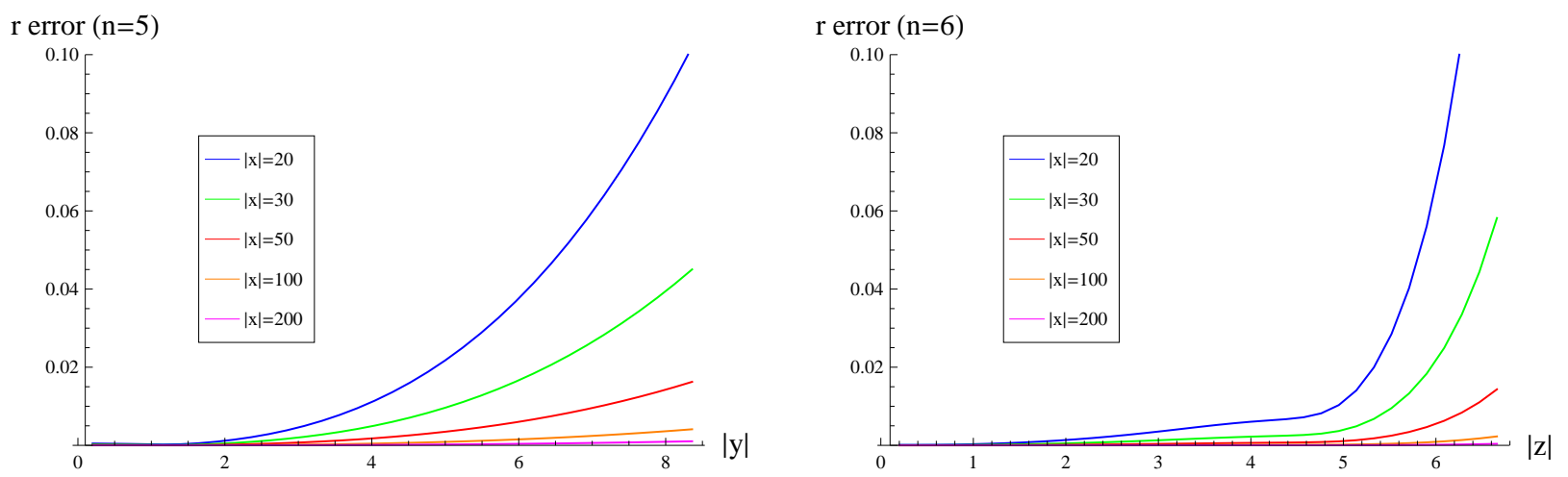

Figure 5: Left picture: relative errors for approximation order $n=5$ and $0<|y|<8$, with $\arg (y)=\pi / 4, z=0.5$, and five increasing values of $|x|$, with $\arg (x)=\pi / 5$. Right picture: relative errors for approximation order $n=6$ and $0<|z|<8$, with $\arg (z)=\pi / 6$, $y=0.2 e^{\mathrm{i} \pi / 5}$, and five increasing values of $|x|$, with $\arg (x)=0$. 